\title{
Anatomical, Cardiovascular, and Blood Gas Parameters in Dogs with Brachycephalic Syndrome
}

\author{
Maria Luíza de Melo Dias' ${ }^{1}$ Carlos Fernando Matias Morris², Bruno Marques Moreti ${ }^{2}$, \\ Arthur Victor do Espírito Santo', Concepta Margaret McManus', \\ Ricardo Miyasaka de Almeida' \& Paula Diniz Galera
}

\begin{abstract}
Background: Brachycephalic syndrome is characterized by increased airflow resistance in upper airways due to the combinations of anatomical deformities such as stenotic nares, elongated soft palate, everted laryngeal saccules, and tracheal hypoplasia. There is little information in recent literature about assessment of anatomical, cardiovascular, and blood gases' parameters of these animals at early stages of the syndrome. The purpose of this study was to characterize and to compare anatomical, cardiovascular, and blood gases' parameters in young brachycephalic or dolichocephalic and mesocephalic dogs. Materials, Methods \& Results: Twenty brachycephalic dogs (BG) and 20 dolichocephalic and mesocephalic dogs (CG), aged up to 5 years, were included in the study. Anatomical abnormalities, systolic (SAP), mean (MAP), and diastolic (DAP) arterial blood pressure were recorded. Blood gas analysis and complete blood counts (CBC) were analyzed. Doppler echocardiography, electrocardiography, ambulatory electrocardiography, and thoracic and cervical radiographs were evaluated. The diameter of the nares in BG was lower when compared to CG $(0.23 \pm 0.08$ versus $0.56 \pm 0.05 \mathrm{~cm}, P<$ 0.001). The $\mathrm{S}_{\mathrm{a}} \mathrm{O}_{2}$ was lower $(P<0.001)$ and MAP was higher in BG $(P=0.05)$. All brachycephalic dogs had sinus arrhythmia (SA), and $15(75 \%)$ of these showed SA higher than $20 \%$ variation between adjacent RR intervals, whereas in CG, 17 (85\%) of animals presented SA and $13(65 \%)$ of these showed SA higher than $20 \%$ variation between adjacent RR intervals. The smallest opening of the nostril, in brachycephalic dogs, was accompanied by a lower $\mathrm{S}_{\mathrm{a}} \mathrm{O}_{2}$ and higher MAP. Results of this study suggest that young brachycephalic dogs have lower $\mathrm{S}_{2} \mathrm{O}_{2}$ and higher MAP when compared with dolichocephalic and mesocephalic dogs.

Discussion: The most relevant aspect of this survey was the confirmation that brachycephalic dogs have lower $\mathrm{S}_{\mathrm{a}} \mathrm{O}_{2}$ and higher MAP when compared to non brachycephalic animals. Few studies correlate upper airway obstruction to cardiovascular and blood gas alterations. Although there were no significant differences between groups for $\mathrm{PaO} 2$ and $\mathrm{AaO}_{2}$, the means for BG were lower and higher, respectively, than CG, showing that obstruction of airways may lead to lower $\mathrm{P}_{\mathrm{a}} \mathrm{O}_{2}$ and higher $\mathrm{A}_{\mathrm{a}} \mathrm{DO}_{2}$. In the present study, brachycephalic animals tended to have higher tHb, PCV, DAP, and MAP, and lower $\mathrm{S}_{\mathrm{a}} \mathrm{O}_{2}$. Even with no significant differences between groups for $\mathrm{tHb}, \mathrm{PCV}$, and DAP, one can assume that young animals may not already have presented such alterations, but as the syndrome progresses, these types of abnormalities tend to develop. Hypoventilation results in significantly lower $\mathrm{S}_{\mathrm{a}} \mathrm{O}_{2}$ in these animals, as observed in this study. All brachycephalic dogs in our study had SA and $2(10 \%)$ showed sinus pauses of $4 \mathrm{~s}$ and $6 \mathrm{~s}$ duration, which demonstrates a stimulated vagal tone. The percentage of cervical soft tissue (CST) in brachycephalic dogs was proposed in an attempt to objectively measure the soft tissue concentrated in the neck region of these animals, which may be considered a risk factor for development of the syndrome. The CST percentage was higher in BG, probably due to the increased amount of adipose tissue in the neck of these animals, fact that can contribute to an increase in tracheal pressure and vascular resistance in the region. Therefore, alterations of brachycephalic syndrome result in significant cardiovascular and blood gas abnormalities in young brachycephalic dogs, as higher MAP and lower $\mathrm{S}_{\mathrm{a}} \mathrm{O}_{2}$.
\end{abstract}

Keywords: brachycephalic syndrome, hypoxemia, hypertension, risk factor. 


\section{INTRODUCTION}

Brachycephalic syndrome is characterized by increased airflow resistance in upper airways because of combinations of anatomical deformities, solely or combined, such as stenotic nares, elongated soft palate, hypoplastic trachea, and nasopharyngeal turbinates [12]. Secondary changes may develop because of the increased turbulence and air resistance and include, in some animals, swelling of the palate and larynx, everted laryngeal saccules, and laryngeal collapse $[4,9,12,15]$.

Elongated soft palate is the most frequent abnormality $(86.3 \%-100 \%)$ followed by stenotic nares $(42.5 \%-85.2 \%)$. Eversion of the laryngeal saccules occurs in $54.1 \%$ to $66 \%$ of dogs, whereas tracheal hypoplasia is the least common finding and affects $8.2 \%$ to $38.2 \%$ of animals $[4,13,14,16,19]$.

Diagnosis is based on clinical signs, as stertorous breathing, inspiratory dyspnea, snoring, coughing, exercise intolerance, cyanosis, and in more severe cases, syncopal episodes [4,14], and physical findings such as detection of stenotic nares and elongated soft palate. However, chest radiographs must also be taken to evaluate tracheal diameter, size of heart chambers, and extent of nasopharynx to assess the soft palate extension [2].

The purpose of this study was to characterize anatomical, cardiovascular and blood gases parameters in brachycephalic animals, by the use of systolic (SAP), mean (MAP), and diastolic (DAP) arterial blood pressure recordings, blood gas analysis, doppler echocardiography, electrocardiography, ambulatory electrocardiography (holter), complete blood count (CBC) as well as thoracic and cervical radiographs, in young dogs aged up to five years.

\section{MATERIALS AND METHODS}

Forty dogs, aged up to 5 years, both males or females, were allocated into two groups: 20 brachycephalic dogs with anatomical changes related to brachycephalic syndrome (Brachycephalic Group = BG) and 20 dolichocephalic and mesocephalic dogs (Control Group = CG), from different breeds. All dolichocephalic and mesocephalic dogs were healthy, whereas brachycephalic ones showed only symptoms related to brachycephalic syndrome. Indocile dogs were not included in the study.
All animals were domiciled and their owners signed a Free and Clear Consent Form. Owners were asked about the dog's age, frequency and intensity of exercise, presence or absence of snoring, coughing, cyanosis, syncope, and open-mouth breathing.

Prior to examination, animals were directed to a quiet room and maintained at a comfortable temperature for $30 \mathrm{~min}\left(21^{\circ} \mathrm{C}\right)$ to provide acclimatization and stress reduction. The diagnosis of stenotic nares was performed by direct inspection without sedation. The elongated soft palate was suspected in animals with snoring.

The exams performed on all animals, chronologically, included: complete physical examination (heart rate, respiratory rate, rectal temperature, mucous membrane color, hydration status, and lymph nodes palpation), measurements of systolic (SAP), mean (MAP), and diastolic (DAP) arterial blood pressure with noninvasive, indirect oscillometric method using cuff corresponding to $40 \%$ of the circumference of the member (PetMAP ${ }^{\circledR}$ Classic) ${ }^{1}$, doppler echocardiography (MyLab ${ }^{\text {TM}} 30$ VET Gold) ${ }^{2}$, electrocardiography $(\mathrm{DL} 650)^{3}$, arterial blood gases analysis (Omni C) ${ }^{4}$, complete blood count (CBC), biochemical profile (Labtest commercial kit) ${ }^{5}$ of renal (urea and creatinine) and hepatic function (alanine transaminase and alkaline phosphatase), chest radiographs (right lateral and ventrodorsal views), cervical radiographs (lateral view with tracheal compression), nasopharynx lateral radiographs (Leonardo DR 1210P) ${ }^{6}$ and ambulatory electrocardiography ${ }^{7}$. Arterial blood samples were obtained by puncture of superficial femoral artery with a $1 \mathrm{~mL}$ syringe containing heparin anticoagulant for blood gas analysis. These samples were placed in a closed cooler with ice and water, and were processed in an automatic analyzer in a period no longer than 2 hours. Five $\mathrm{mL}$ of venous blood samples were obtained by puncture of external jugular vein to complete haemogram, as well as biochemical profile of renal and hepatic functions were taken to ascertain dogs' health.

The opening of nostrils was measured in all animals from both groups by caliper gauge (Disma 150 $\left.\mathrm{mm} 6^{\prime}\right)^{8}$, and this was defined as the distance between nasal septum and dorsal nasal cartilage.

The segmental percentage of cervical soft tissue (CST) was proposed and calculated based on lateral cervical radiographs of animals from both groups, where the sum of the diameter of the fourth cervical 
vertebra (VertD) and the diameter of the tracheal lumen (TD) was subtracted from the total cervical vertebrae diameter (CervD), as defined by the following equation: $\operatorname{CST}(\%)=[$ CervD $-($ VertD + TD $) / C e r v D] \times 100$. The fourth cervical vertebra $(\mathrm{C} 4)$ was the standard reference (Figure 1).

Cardiac area was measured based on the right chest radiography through the vertebral scale system (VHS - Vertebral Heart Score) that compared heart dimensions to the sum of thoracic vertebrae length, starting at the anterior edge of the 4th thoracic vertebra [18].

Data were analyzed using SAS $^{\text {TM9 }}$ (Statistical Analysis System, v. 9.3) program. For frequency analysis a $\chi^{2}$ test was used and comparison between groups for parametric data used Duncan test. Variables were considered significant when $P<0.05$. Pearson correlations and principal components were calculated to investigate the relationships between the variables obtained.

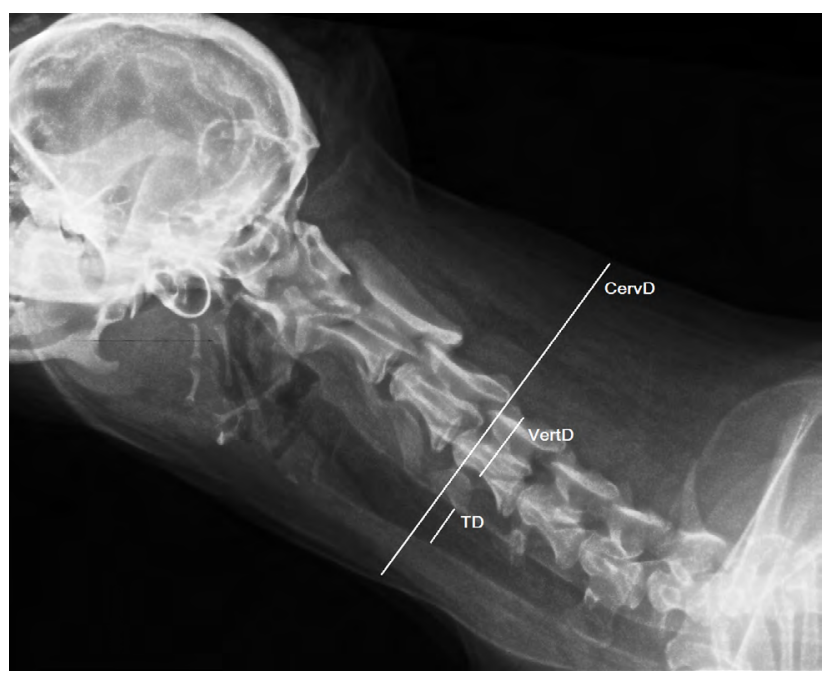

Figure 1. Lateral cervical radiographic image of a 2-year-old Shih Tzu from $B G$ used to measure the segmental percentage of cervical soft tissue (TD, diameter of the tracheal lumen; VertD, diameter of the fourth cervical vertebra; CervD, total cervical diameter).

\section{RESULTS}

The BG was composed of: 12 (60\%) Shih Tzus, 4 (20\%) French Bulldogs, 3 (15\%) Pugs, and 1 (5\%) English Bulldog. The mean age \pm standard deviation (SD) of these animals was $29.20 \pm 19.13$ months. From the 20 animals included in this group, $6(30 \%)$ were $\leq 1$ year old, $8(40 \%)$ were $>1$ to $\leq 3$ years old and 6 dogs $(30 \%)$ were $>3$ to $\leq 5$ years old. The mean \pm SD body mass was $8.36 \pm 3.74 \mathrm{~kg}$, with a range body weight of 2.6 to $16.2 \mathrm{~kg}$.
The CG was composed of 9 (45\%) mixed breed dogs (MBD), 4 (20\%) Dachshunds, 3 (15\%) Shetland Shepherds, 2 (10\%) German Spitz, 1 (5\%) Bichon Frisé, and $1(5 \%)$ Beagle. The mean age \pm SD of these animals was $32.80 \pm 19.93$ months. Of these $20 \mathrm{dogs}$, $7(35 \%)$ were $\leq 1$ year old, $4(20 \%)$ were $>1$ to $\leq 3$ years old, and 9 dogs (45\%) were $>3$ to $\leq 5$ years old. The mean \pm SD body mass was $9.78 \pm 4.28 \mathrm{~kg}$, with a range body weight of 3.6 to $17.4 \mathrm{~kg}$.

According to owners of brachycephalic dogs, the presence of stertorous inspiration was observed in $18 \mathrm{dogs}(90 \%)$, sporadic episodes of cough were observed in 5 dogs (20\%), and 1 dog (5\%) presented cyanosis after exercise. Syncopal episodes were not reported in any animal. Sixteen $(80 \%)$ of these dogs were open mouth breathers, $5(25 \%)$ of those were open mouth breather even at rest, these being $3(41 \%)$ Shih tzus, 1 (33\%) Pug, and 1 (25\%) French Bulldog.

When examined for anatomic components of brachycephalic syndrome, 18 dogs $(90 \%)$ had elongated soft palate, 9 dogs $(45 \%)$ had stenotic nares, and 7 dogs (35\%) presented both changes. Three of these (100\%) were Pugs, 2 (16.6\%) were Shih Tzus, and 2 $(50 \%)$ were French Bulldogs. As the animals were not sedated, their laryngeal saccules were not inspected. No animal showed evidence of tracheal hypoplasia. Cervical trachea collapse was not observed in any animal, as well as in lateral radiographs with cervical compression. Dogs of CG did not show any anatomical abnormalities related to brachycephalic syndrome, and the owners did not report clinical changes.

The mean \pm SD of temperature, heart rate $(\mathrm{HR})$ and respiratory rate $(f)$ of $\mathrm{BG}$ were $39.0 \pm 0.45^{\circ} \mathrm{C}$, $116 \pm 24$ beats per min, and $36 \pm 10$ breaths per min, respectively, whereas for $\mathrm{CG}$, these parameters were $38.9 \pm 0.62^{\circ} \mathrm{C}, 109 \pm 22.7$ beats per min, and $33 \pm 7.26$ breaths per min, respectively.

The mean \pm SD of opening of the nostril in $\mathrm{BG}$ and $\mathrm{CG}$ were $0.23 \pm 0.08 \mathrm{~cm}$ and $0.56 \pm 0.05 \mathrm{~cm}$, respectively. The nasal opening in $\mathrm{BG}$ was significantly lower $(P<0.001)$. The mean $\pm \mathrm{SD}$ of the diameter of cervical trachea for BG was $1.05 \pm 0.10 \mathrm{~cm}$, whereas for CG it was $1.28 \pm 0.19 \mathrm{~cm}(P>0.05)$.

The findings related to SAP, MAP, DAP, and blood gases analysis are represented as means \pm SD in Table 1. The MAP was higher in BG $(P=0.05)$, and there were no statistical differences between groups for SAP and DAP $(P=0.71$ and 0.07 , respectively). 
Arterial hemoglobin saturation $\left(\mathrm{S}_{\mathrm{a}} \mathrm{O}_{2}\right)$ was lower in $\mathrm{BG}(P<0.001)$. The $\mathrm{pH}$ was within the normal range, with no significant differences between groups. No significant differences between groups were found for arterial bicarbonate concentration $\left(\left[\mathrm{HCO}^{-}\right]_{\mathrm{a}}\right)$ and partial pressure of arterial carbon dioxide $\left(\mathrm{P}_{\mathrm{a}} \mathrm{CO}_{2}\right)$. The anion gap was lower in BG $(P=0.03)$. There were no statistical differences between groups for hemoglobin concentration $(\mathrm{tHb})$ and partial pressure of arterial oxygen $\left(\mathrm{P}_{\mathrm{a}} \mathrm{O}_{2}\right)$, although the means are higher and lower, respectively, in BG. There were no significant differences between groups for alveolararterial oxygen gradient $\left(\mathrm{A}_{\mathrm{a}} \mathrm{DO}_{2}\right), \mathrm{tHb}$, and packed cell volume (PCV).

Doppler echocardiography assessment of pulmonary artery flow velocity pattern was lower in BG $(P$ $=0.005)$ than in $\mathrm{CG}$, with mean $\pm \mathrm{SD}$ of $77.75 \pm 12.98$ $\mathrm{cm} / \mathrm{s}$ and $95.92 \pm 20.35 \mathrm{~cm} / \mathrm{s}$, respectively. Detection of segmental myocardial wall contraction by M-mode in cross-sectional planes of atrioventricular transvalvular flows and exit routes appliances, using pulsed Doppler, and of the dimensions of cardiac chambers, showed no abnormalities in any of the animals.

Electrocardiogram (ECG) showed a higher presence of migratory pacemaker in BG $(P=0.02)$, which was exhibited in $14(70 \%)$ and $6(30 \%)$ dogs in BG and CG, respectively. All brachycephalic dogs had sinus arrhythmia (SA) and 15 (75\%) of these dogs had a SA variation higher than $20 \%$ between adjacent R-R intervals. In CG, 17 (85\%) dogs had SA and 13 (65\%) of these exhibited variation higher than $20 \%$ between adjacent R-R intervals.

During ambulatory electrocardiographic examination, 2 dogs (10\%) of BG presented sinus pauses of $4 \mathrm{~s}$ and $6 \mathrm{~s}$ duration. During the exam, the maximum, minimum, and mean $\mathrm{HR}$ in $\mathrm{BG}$ were 252 $\pm 22,48 \pm 18$, and $83 \pm 14 \mathrm{bpm}$, respectively. In CG, these parameters were $247 \pm 21,37 \pm 12$, and $72 \pm$ $8 \mathrm{bpm}$, respectively. The mean HR was significantly higher in BG $(P=0.008)$.

The mean \pm SD of segmental percentage of cervical soft tissue was $77.57 \pm 3.80 \%$ and $71.91 \pm$ $5.06 \%$ in $\mathrm{BG}$ and $\mathrm{CG}$, respectively, with significant difference between groups $(P=0.02)$. There was no significant correlation between variables such as SAP, MAP, DAP, HR, and $\mathrm{f}$. The mean \pm SD of VHS in BG and $C G$ was $9.60 \pm 0.73$ and $9.45 \pm 0.6$, respectively, with no significant differences between groups.

\section{DISCUSSION}

Amongst the breeds studied, results in the present study were similar to those previously described in literature [4], with Shih Tzu as the most prevalent breed in this study, probably due to the local growing popularity of the breed.

Consistent with the literature, inspiratory stertor was the most frequent clinical sign observed [4,17], associated with an excess of soft tissues in upper airway, such as an elongated soft palate [16]. Similar to previous reports $[4,13,14,16]$, an elongated soft palate was observed more frequently than stenotic nares. Although tracheal hypoplasia is reported with a frequency varying from $8.2 \%$ to $38.2 \%[4,13]$, this abnormality was not encountered in animals from this study.

The most relevant aspect of this survey was the confirmation that brachycephalic dogs have lower $\mathrm{S}_{\mathrm{a}} \mathrm{O}_{2}$ and higher MAP when compared to non brachycephalic animals. Few studies correlate upper airway obstruction to cardiovascular and blood gas alterations. In a study performed by Hoareau et al. [8] on 11 brachycephalic dogs with mean age \pm SD of $43 \pm$ 19 months, $\mathrm{P}_{\mathrm{a}} \mathrm{O}_{2}$ was lower but there was no statistical difference between $\mathrm{A}_{\mathrm{a}} \mathrm{DO}_{2}$, and higher tHb and PCV. This showed that hypoventilation results in lower $\mathrm{P}_{\mathrm{a}} \mathrm{O}_{2}$ and a compensatory mechanism may be put in place to maintain normal arterial oxygen content, by stimulating the production of red blood cells, as a result of chronic hypoxia in these animals. In our study, lack of a significant difference in $\mathrm{P}_{\mathrm{a}} \mathrm{O}_{2}$ and $\mathrm{tHb}$ between groups may be associated, in part, to the young age of the animals (mean $=29.20 \pm 19.13$ months), assuming that they do not have chronic inflammation of upper and bronchial airways, caused by recurrent barotrauma that contributes to lower $\mathrm{P}_{\mathrm{a}} \mathrm{O}_{2}$ [8].

Mean $\mathrm{P}_{\mathrm{a}} \mathrm{CO}_{2}$ was higher in $\mathrm{BG}$, but no statistically significant difference between groups. This may be associated with hypoventilation suffered by these animals due to upper airway obstruction. Hoareau et al. [8] compared groups of brachycephalic dogs and concluded that dogs with higher $\mathrm{P}_{\mathrm{a}} \mathrm{CO}_{2}$ are significantly older than those with lower $\mathrm{P}_{\mathrm{a}} \mathrm{CO}_{2}$. Additionally, there was no difference between young brachycephalic and non brachycephalic dogs, similar to our study. Age seems to be an important factor in blood gas analysis and is associated with decreased compliance or increased airway resistance [8]. The mean $\left[\mathrm{HCO}_{3}^{-}\right]_{\mathrm{a}}$ was also higher in brachycephalic animals when compared 
to CG, but no statistically significant. This increase may be attributed to metabolic compensatory responses to maintain a normal $\mathrm{pH}$ range [8].

Although there were no significant differences between groups for $\mathrm{P}_{\mathrm{a}} \mathrm{O}_{2}$ and $\mathrm{A}_{\mathrm{a}} \mathrm{DO}_{2}$, the means for $\mathrm{BG}$ were lower and higher, respectively, than $\mathrm{CG}$, showing that obstruction of airways may lead to lower $\mathrm{P}_{\mathrm{a}} \mathrm{O}_{2}$ and higher $\mathrm{A}_{\mathrm{a}} \mathrm{DO}_{2}$. Hoareau et al. [8] found a significant lower $\mathrm{P}_{\mathrm{a}} \mathrm{O}_{2}$ in brachycephalic dogs associated with airway.

Humans patients with conformational abnormalities that lead to increased airway resistance, such as constrictions in the upper airways, obesity, and large tongue, have higher risk of developing obstructive sleep apnea (OSA), characterized by the complete upper airway collapse during sleep that can result in intermittent hypoxemia and systemic hypertension [6]. It is reasonable to assume that brachycephalic dogs may also have higher risk for OSA. English Bulldogs have been used as a spontaneous model for humans with OSA, but it is still unknown whether dogs with brachycephalic syndrome also have an increased risk for hypertension [8].

The mean SAP, DAP, and MAP were higher in $\mathrm{BG}$, but only MAP was statistically significant. Hoareau et al. [8] also demonstrated increased MAP in brachycephalic animals. The specific mechanisms that cause hypertension in humans with OSA have not been clearly elucidated, but it has been proposed that intermittent hypoxia, persistent sympathetic activation of renin-angiotensin-aldosterone system [6], systemic inflammation, and changes in intrathoracic pressure contribute to hypertension $[5,10]$. Hypoxemia may also occur in younger animals with brachycephalic syndrome, leading to an increase in their MAP [8].

In the present study, brachycephalic animals tended to have higher tHb, PCV, DAP, and MAP, and lower $\mathrm{S}_{\mathrm{a}} \mathrm{O}_{2}$, as demonstrated on principal component analysis. Even with no significant differences between groups for $\mathrm{tHb}, \mathrm{PCV}$, and DAP, one can assume that young animals may not already have presented such alterations, but as the syndrome progresses, these types of abnormalities tend to develop. Hypoventilation results in significantly lower $\mathrm{S}_{\mathrm{a}} \mathrm{O}_{2}$ in these animals, as observed in this study.

Mean pulmonary artery velocity flow was significant lower in BG $(P=0.005)$, but both groups had this parameter within the reference value (76-122 $\mathrm{cm} / \mathrm{s}$ [1]); therefore, there is no evidence of changes in pulmonary pressure in early stages of the syndrome.

Sinusal arrhythmia is a common and normal rhythm in dogs and is associated with increased parasympathetic activity in sinoatrial node [11]. All brachycephalic dogs in our study had SA and two (10\%) showed sinus pauses of $4 \mathrm{~s}$ and $6 \mathrm{~s}$ duration, which demonstrates a stimulated vagal tone. Migratory pacemaker is a cyclical change in $\mathrm{P}$ wave morphology that also can be associated with increased vagal tone and is normal and common in dogs [11]. This electrocardiographic find was present in $70 \%$ of brachycephalic dogs in our study. Doxey \& Boswood [3] reported that brachycephalic dogs have vasovagal tonus index greater than non brachycephalic dogs; nevertheless, the cause for this increase remains unknown.

The most significant risk factor for developing OSA in humans appears to be upper-body fat accumulation, particularly in the neck [5]. The percentage of cervical soft tissue (CST) in brachycephalic dogs was proposed in an attempt to objectively measure the soft tissue concentrated in the neck region of these animals, which may be considered a risk factor for development of the syndrome. The CST percentage was higher in $\mathrm{BG}$, probably due to the increased amount of adipose tissue in the neck of these animals, fact that can contribute to an increase in tracheal pressure and vascular resistance in the region.

Recent studies revealed that brachycephalic dogs with chronic airway obstruction might have pro-inflammatory biomarkers, suggesting that brachycephalic syndrome also results in systemic alterations through the production of inflammatory mediators [15].

\section{CONCLUSIONS}

Alterations of brachycephalic syndrome resulting in significant cardiovascular and blood gas abnormalities in young brachycephalic dogs, as higher MAP and lower $\mathrm{S}_{\mathrm{a}} \mathrm{O}_{2}$. Therefore, blood gas analysis, ECG, and arterial pressure measurements are fundamental for determining the health status of these animals and early identification of cardiovascular and blood gas alterations in animals with brachycephalic syndrome emphasizes the importance of treatment as early as possible, besides being accessible and low-cost tests. 


\section{MANUFACTURERS}

${ }^{1}$ PetMAP®. Tampa, FL, USA.

${ }^{2}$ Esaote Healthcare do Brasil. São Paulo, SP, Brazil.

${ }^{3}$ Delta Life. São José dos Campos, SP, Brazil.

${ }^{4}$ Roche Diagnostics. São Paulo, SP, Brazil.

${ }^{5}$ Labtest Diagnóstica SA. Belo Horizonte, MG, Brazil.

${ }^{6}$ Macrotec. Cotia, SP, Brazil.

${ }^{7}$ Medata Inc. Irvine, CA, USA.

${ }^{8}$ Disma Ferramentas. São Paulo, SP, Brazil.

${ }^{9}$ Statistical Analysis System. Cary, NC, USA.
Ethical approval. All procedures, treatments and animal care were approved by the Ethics Committee for Animal Use (CEUA) of the University of Brasilia (protocol no. 79264/2013).

Declaration of interest. The authors report no conflicts of interest. The authors alone are responsible for the content and writing of paper.

\section{REFERENCES}

1 Boon J.A. 2006. Manual of Veterinary Echocardiography. 3rd edn. Ames: Wiley-Blackwell, 478p.

2 Bowlt K. \& Moore A.H. 2009. Surgery of the upper respiratory tract. Part 2. Brachycephalic obstructive airway syndrome (BOAS). UK Veterinary. 14(8): 1-6.

3 Doxey S. \& Boswood A. 2004. Differences between breeds of dog in a measure of heart rate variability. Veterinary Record. 154(23): 713-717.

4 Fasanella F.J., Shivley J.M., Wardlaw J.L. \& Givaruangsawat S. 2010. Brachycephalic airway obstructive syndrome in dogs: 90 cases (1991-2008). Journal of the American Veterinary Medical Association. 237(9): 1048-1051.

5 Fava C., Montagnana M., Favaloro E.J., Guidi G.C. \& Lippi G. 2011. Obstructive sleep apnea syndrome and cardiovascular diseases. Seminars in Thrombosis and Hemostasis. 37(3): 280-297.

6 Foster G.E., Hanly P.J., Ahmed S.B., Beaudin A.E., Pialoux V. \& Poulin M.J. 2010. Intermittent hypoxia increases arterial blood pressure in humans through a rennin-angiotensin system-dependent mechanism. Hypertension. 56(3): 369-377.

7 Haskins S., Pascoe P.J., Ilkiw J.E., Fudge J., Hopper K. \& Aldrich J. 2005. Reference cardiopulmonary values in normal dogs. Comparative Medicine. 55(2): 156-161.

8 Hoareau G.L., Jourdan G., Mellema M. \& Verwaerde P. 2012. Evaluation of arterial blood gases and arterial blood pressures in brachycephalic dogs. Journal of Veterinary Internal Medicine. 26(4): 897-904.

9 Koch D.A., Arnold S., Hubler M. \& Montavon P.M. 2003. Brachycephalic syndrome in dogs. Compendium Vetlearn. 25(1): 48-55.

10 Kohler M. \& Stradling J.R. 2010. Mechanisms of vascular damage in obstructive sleep apnea. Nature Reviews Cardiology. 7(12): 677-685.

11 Martin M. 2007. Small Animal ECGs: an introductory guide. 2nd edn. Oxford: Wiley-Blackwell, 136p.

12 Meola S.D. 2013. Brachycephalic Airway Syndrome. Topics in Companion Animal Medicine. 28(3): 91-96.

13 Poncet C.M., Dupre G.P., Freiche V.G., Estrada M.M., Poubanne Y.A. \& Bouvy B.M. 2005. Prevalence of gastrointestinal tract lesions in 73 brachycephalic dogs with upper respiratory syndrome. Journal of Small Animal Practice. 46(6): 273-279.

14 Poncet C.M., Dupre G.P., Freiche V.G. \& Bouvy B.M. 2006. Long-term results of upper respiratory syndrome surgery and gastrointestinal tract medical treatment in 51 brachycephalic dogs. Journal of Small Animal Practice. 47(3): 137-142.

15 Rancan L., Romussi S., Garcia P., Albertini M., Vara E.\& Sanchez De La Muela M. 2013. Assessment of circulating concentrations of proinflammatory and anti-inflammatory cytokines and nitric oxide in dogs with brachycephalic airway obstruction syndrome. American Journal of Veterinary Research. 74(1): 155-160.

16 Riecks T.W., Birchard S.J. \& Stephens J.A. 2007. Surgical correction of brachycephalic syndrome in dogs: 62 cases (1991-2004). Journal of the American Veterinary Medical Association. 230(9): 1324-1328.

17 Roedler F.S., Pohl S. \& Oechtering G.U. 2013. How does severe brachycephaly affect dog's lives? Results of a structured preoperative owner questionnaire. The Veterinary Journal. 198(3): 606-610.

18 Tilley L.P. \& Goodwin J.K. 2002. Manual de Cardiologia para Cães e Gatos. São Paulo: Roca, 489p.

19 Torrez C.V. \& Hunt G.B. 2006. Results of surgical correction of abnormalities associated with brachycephalic airway obstruction syndrome in dogs in Australia. Journal of Small Animal Practice. 47(3):150-154. 